\title{
Modelo para el análisis de la estrategia de operaciones de las empresas exportadoras del sector metalmecánico del triángulo del café de Colombia
}

\author{
Luis I. López ${ }^{1}$, Carlos E. Marulanda ${ }^{1,3}$ y Jhon Salinas-Ávila ${ }^{2}$ \\ (1) Departamento de Administración, Facultad de Administración, Universidad Nacional de Colombia Sede Manizales, \\ Carrera 27 No.64-60, Manizales, Caldas-Colombia. (correo-e: lilopezv@unal.edu.co, cemarulandae@unal.edu.co) \\ (2) Departamento de Economía y Administración, Facultad de Ciencias Jurídicas y Sociales, Universidad de Caldas, Sede \\ Palogrande, Carrera 23 No. 58-65, Manizales, Caldas-Colombia. (correo-e: jhon.salinas@ucaldas.edu.co) \\ (3) Departamento de Sistemas e informática, Facultad de Ingeniería, Universidad de Caldas, Calle 65 N0. 26-10, \\ Manizales, Caldas-Colombia. (correo-e: carlose@ucaldas.edu.co)
}

Recibido Mar. 31, 2021; Aceptado Jun. 7, 2021; Versión final Jun. 30, 2021, Publicado Dic. 2021

\begin{abstract}
Resumen
El objetivo del presente estudio es validar estadísticamente un modelo para el análisis de la estrategia de operaciones de las empresas exportadoras del sector metalmecánico del triángulo del café de Colombia. Se desarrolla un constructo teórico a partir de modelos de análisis existentes y a partir del juicio de expertos para determinar las categorías y variables requeridas. El uso de la técnica estadística de correlación permitió validar con resultados altamente significativos que el modelo fue construido con las prioridades competitivas adecuadas para el sector y tipo de empresas seleccionadas. Se obtuvo un modelo de estrategia de operaciones integral que evalúa siete categorías: costo, calidad, flexibilidad de proceso, flexibilidad de producto, entrega, servicio y medio ambiente. Se concluye que este modelo está adaptado a las necesidades de las empresas estudiadas y que podrá ser utilizado en otras similares.
\end{abstract}

\section{Operation strategy analysis model for export companies in the metal-mechanic sector from the Colombian coffee triangle}

\begin{abstract}
The main objective of the present study is to statistically validate an operation strategy analysis model for metal-mechanic export companies from the Colombian coffee triangle. A theoretical construct is developed based on existing analysis models and expert judgment to determine the categories and variables required. The use of the statistical technique of correlational allowed to validate with highly significant results that model was built with the appropriate competitive priorities for the sector and type of the companies selected. A comprehensive operations strategy model was obtained that evaluates seven categories: cost, quality, process flexibility, product flexibility, delivery, service, and environment. It is concluded that the model is adapted to the needs of the companies assessed and that it can be applied to evaluate similar companies.
\end{abstract}

Keywords: process modeling; operations strategy; metalworking sector; export 


\section{INTRODUCCIÓN}

La situación de la industria manufacturera, a partir de la internacionalización de la economía, se ha vuelto más compleja y exige por parte de las empresas cambios sustanciales en su estrategia competitiva y de operaciones. La economía cada vez más integrada, ha obligado a las empresas a aumentar la productividad, expandirse a nuevos mercados, adoptar nuevas tecnologías, atraer y asegurar la fuerza laboral (Korsakienè et al., 2017), pues, la realidad mundial es de intensidad en la rivalidad de las empresas por el mercado, impulsado esto por la disminución de los aranceles, tratados de libre comercio y el fortalecimiento de regiones económicas, lo que se ha denominado "nuevo regionalismo" con la creación de tratados liderados por las grandes transnacionales, que compiten con la visión de multilateralismo de la Organización Mundial del Comercio (Guerra-Borges, 2008).

Las empresas de fabricación están cada vez más frente a diversas y crecientes presiones debido a la globalización, la tecnología, a mercados más sofisticados, así como a cambios en las preferencias del consumidor y la competencia global, entre otros. Las compañías de categoría mundial desarrollan procesos y métodos de excelencia, buscando competir en todas las dimensiones, creando robustas organizaciones de producción, con una cultura de productividad y fuente de estrategia competitiva (Martínez-Sánchez et al., 2019; Andrade et al., 2019). Esta situación ha generado un escenario tan competitivo que las empresas tienen que buscar nuevos procesos, nuevos materiales, nuevos proveedores, nuevos diseños de fabricación y nuevos canales para ofrecer sus productos y servicios a precios competitivos (Dangayach, 2001). Bajo este contexto, la estrategia de operaciones emerge como respuesta a esta necesidad, y por ello, la función de operaciones/producción debe concebirse estratégicamente como una fuente de ventaja competitiva.

La estrategia de operaciones se asienta sobre capacidades o prioridades competitivas: costo, calidad, flexibilidad y tiempo de entrega. Ser capaz de competir en el futuro con éxito, podría requerir la identificación, desarrollo y explotación de nuevas capacidades estratégicas tales como la innovación, con el fin de diferenciarse de la mayor parte de los competidores (Größler, 2010). Otra es la responsabilidad ambiental, la cual se suma a las prioridades competitivas, de hecho, las empresas en el nuevo siglo están prestando más atención a los aspectos ambientales con el propósito de mejorar su competitividad sostenible a través de prácticas verdes y ajustadas (lean) (Zhan et al., 2018). De este modo, el interés en las prioridades competitivas de la estrategia de operaciones entra a jugar un papel importante como parte de la estrategia de operaciones y fuente de ventajas competitivas (Chatha y Butt, 2015). Aunque escapa del alcance del presente trabajo, es importante señalar el trabajo de De la Hoz et al., (2016), quienes presentan una importante perspectiva de factores clave en la medición del potencial exportador. En particular, proponen evaluar la empresa en las siguientes dimensiones clave: financiera, procesos internos, aprendizaje y crecimiento, clientes y mercado.

Lo anterior, obliga a las empresas a realizar una planeación estratégica de la manufactura/operaciones, para superar a sus rivales, con un enfoque en el análisis del desempeño de la organización y sus operaciones, a partir de la interfuncionalidad del trabajo, máquinas, procesos, materiales, productos, clientes y proveedores (Göleç, 2015). De igual manera, la empresa debe conocer cuál es su posición competitiva en cuanto a su estrategia de operaciones, con el fin de construir nuevas capacidades y así garantizar su supervivencia y crecimiento. Ante esta realidad, las empresas reaccionan de manera diferente y la calidad de su respuesta repercute en su posición competitiva, la cual debe enfocarse en el logro de los objetivos organizacionales de supervivencia, crecimiento y rentabilidad. Las prioridades competitivas que integran la estrategia de operaciones, han ido evolucionando a través del tiempo y se derivan de los diversos procedimientos que hacen parte del proceso productivo y de las áreas en que debe centrarse la gestión de operaciones para crear ventajas y generar productos que apoyen la estrategia competitiva (Avella et al., 1999).

Sáenz (2005) define la estrategia de operaciones como un plan o una visión de largo plazo para la función de operaciones, la cual se debe integrar con la estrategia de la organización. Este plan debe contener la misión, objetivos, políticas y competencias distintivas. Es decir, la estrategia de operaciones debe responder tanto a la estrategia de la organización como a la consistencia interna en la toma de decisiones respecto a las operaciones a través del tiempo. Así mismo, dicha estrategia es el proceso que procura alinear las operaciones con la estrategia de la organización y con las estrategias de otras áreas, tales como las de marketing y finanzas, a fin de apoyar la estrategia de negocio de la empresa. Bajo esta misma línea, (Zhao y Lee, 2009), explican que la estrategia de operaciones es un plan que establece la visión y la dirección general para la toma de decisiones en materia de operaciones. La estrategia de operaciones suele definirse en función de objetivos clave como la calidad, la entrega, la flexibilidad, el costo, el servicio y la innovación. Además, la globalización, el aumento de las expectativas de los clientes y los avances en la tecnología de la información han provocado cambios drásticos en las prioridades competitivas de las empresas que las obliga a introducir mejoras masivas en múltiples dimensiones de sus capacidades operativas.

Lobo et al., (2019a, 2019b), precisan que la estrategia de operaciones puede definirse como el desarrollo de la competitividad basada en la función de producción, la cual ayuda a alcanzar los objetivos competitivos a 
largo plazo. La estrategia de operaciones debe considerar cómo las necesidades del mercado y las capacidades de fabricación pueden combinarse mediante la estrategia competitiva en un mercado dinámico e impredecible para mantener el rendimiento competitivo. Es así como una estrategia de producción satisfactoria debe responder cuestionamientos en cuanto a la coherencia con las exigencias del entorno ( $¿$ en qué condiciones económicas y tecnológicas intenta la empresa ejecutar su estrategia?), las exigencias de la competencia (¿cuáles son los puntos fuertes y débiles de los competidores? ¿Qué intentan hacer?), la estrategia de la empresa (¿Qué intenta hacer la empresa?) y el ciclo de vida del producto (¿En qué punto del ciclo de vida se encuentran las ofertas de la empresa?) (Badri et al., 2000).

En consecuencia, "la estrategia de operaciones se convierte en un elemento fundamental para la planeación y ejecución de acciones conducentes a la optimización de los recursos de la compañía, como una forma de lograr posicionar al ente empresarial de manera competitiva en el turbulento mundo de los negocios de la sociedad globalizada" (León, 2020). Agrega el mismo autor que: "Las prioridades competitivas y valores corporativos sirven de norte a la hora de planificar la estrategia operativa, la cual debe estar en consonancia con ellos. Las estrategias pueden ser de muy diversa índole y pueden hacer hincapié en aspectos competitivos diferentes. Las áreas de decisión sobre las que inciden abarcan ámbitos diversos. El propósito de la estrategia de operaciones es lograr ventaja competitiva, es decir, posicionamiento en la mente de los clientes". La función de operaciones de los sectores manufactureros están condicionadas por una serie de retos, dadas las amenazas del entorno y competidores con capacidades de producción que han desnudado una serie de debilidades estructurales que ponen en riesgo su permanencia en el mercado. Desde la década de los años 70 del siglo pasado, con la irrupción del modo de producción japonés, donde la función de operaciones tenía poca cabida en la visión estratégica de la organización, se requiere un cambio de rumbo, desde la perspectiva estratégica y competitiva de la organización (Patil et al., 2015). Así, la estrategia de operaciones debe enlazar las acciones desde su formulación estratégica con el desempeño de la empresa. Es decir, la formulación estratégica exige análisis de alternativas, inducidas por los rápidos cambios tecnológicos y de procesos en un entorno competitivo global donde existen clientes más informados y exigencias mayores en lo ambiental y social. En la actualidad, se considera que cualquier organización puede otorgar una importancia estratégica a la función de producción que sea consistente con la estrategia de negocio para la consecución de una ventaja competitiva (Díaz y Martín, 2007).

Arafa y EIMaraghy (2011) detallan la estrategia de operaciones como un patrón de decisiones, tanto estructurales como infraestructurales, que determinan la capacidad de un sistema de fabricación para cumplir con un conjunto de objetivos que se ajustan a las metas comerciales de la empresa. En este sentido, la estrategia de operaciones requiere ser evaluada como generadora de ventajas competitivas y la jerarquización de las prioridades se convierte en un insumo de la propia estrategia, para lo cual es necesario crear un modelo que evalué la estrategia de operaciones, jerarquice las prioridades competitivas, identifique las competencias necesarias que coadyuven a la estrategia de operaciones y la conviertan en fuente de supervivencia y crecimiento. Dado el dinamismo del entorno, los ajustes estratégicos hacen que las empresas re-prioricen las áreas de toma de decisiones, definan las prioridades competitivas, reafirmen el costo de fabricación, la flexibilidad, la calidad y el desempeño de entrega (Thürer et al., 2014).

Para dar respuesta a los planteamientos de los párrafos anteriores relacionadas con las presiones del entorno que obligan a las organizaciones a adaptarse con mayor agilidad a los cambios que ocurren en él, se plantea un modelo que considera las prioridades competitivas como enlace entre la estrategia de producción y la estrategia empresarial en términos de costo, calidad, tiempo de entrega y flexibilidad (Wheelwright,1978), innovación y responsabilidad ambiental (Dangayach y Deshmukh, 2001). Igualmente, es necesario tener en cuenta que las empresas reaccionan a cambios en el entorno, transformándose en búsqueda de mantener su competitividad y logrando acumular mayores capacidades y competencias. Lo que hace posible plantear el modelo con base en siete prioridades competitivas, a saber: costo, calidad, flexibilidad de proceso, flexibilidad de producto, entrega, servicio y medio ambiente. Dichas prioridades competitivas se llevan al diseño de un modelo de análisis de estrategia de operaciones considerando las categorías y variables tal como se puede observar en la tabla 1. Y como aporte del trabajo de investigación, se evalúa la capacidad explicativa de los resultados y la posición competitiva derivada de la acumulación de recursos y capacidades al interior de la empresa, así como la definición de elementos críticos de éxito, dada la importancia "que las empresas midan y evalúen" (De La Hoz et al., 2016)

\section{METODOLOGÍA}

La investigación se enfocó en la comprobación, considerando el desarrollo de un modelo de análisis de la estrategia de operaciones, el cual se aplicó a empresas y organizaciones medianas y grandes del sector metalmecánico del triángulo del café de Colombia. Así, en primer lugar, se realizó una profunda revisión de la literatura para analizar e identificar las principales variables que podrían ser susceptibles de incluir en el modelo de evaluación de la estrategia de operaciones de una empresa. Esto, con el fin de garantizar la validez y efectividad del modelo. En segundo lugar, se consultaron académicos en el área de estudio, cuyas 
sugerencias se incorporaron al modelo para que este fuera más aplicable a las empresas objeto de análisis. Así mismo, se les pidió a estos expertos que describieran las interacciones que se presentan entre los diferentes factores que tienen incidencia en la estrategia de operaciones de las empresas. Por último, considerando el diseño del modelo de análisis de la estrategia de operaciones, se realizó un análisis correlacional para encontrar la dependencia y/o influencia entre las variables. Con base en esta metodología se construyó y validó dicho modelo, el cual se compone de 7 categorías, 21 variables de análisis y 33 indicadores. La escala de medida fue Likert, con un rango de 1 a 5, donde: 1 es deficiente y 5 excelente.

Tabla 1: Variables

\begin{tabular}{|l|l|l|}
\hline Categorías & Variables & Definición \\
\hline Costo & Bajo costo & Capacidad para producir con alta productividad \\
\hline \multirow{4}{*}{ Calidad } & Desempeño & Características principales de operación del producto \\
\cline { 2 - 3 } & Rasgos distintivos & Características que complementan el producto básico \\
\cline { 2 - 3 } & Confiabilidad & Probabilidad de falla o mal funcionamiento en un período dado \\
\cline { 2 - 3 } & Conformidad & Grado en que el producto satisface las especificaciones de diseño \\
\cline { 2 - 3 } & Durabilidad & Extensión de la vida económica del producto \\
\hline \multirow{4}{*}{$\begin{array}{l}\text { Flexibilidad } \\
\text { de proceso }\end{array}$} & Mezcla & Capacidad de producir más de un producto a la vez \\
\cline { 2 - 3 } & Gama & Capacidad de producir una amplia variedad de productos \\
\cline { 2 - 3 } & Máquina & Capacidad para cambiar de un conjunto de partes a otro \\
\cline { 2 - 3 } & Modificación & Capacidad para incorporar cambios en los diseños del producto \\
\cline { 2 - 3 } & Recorrido & Capacidad para alterar la secuencia de las máquinas \\
\hline \multirow{3}{*}{$\begin{array}{l}\text { Flexibilidad } \\
\text { de producto }\end{array}$} & Expansión & Capacidad para ampliar o rediseñar el sistema fácilmente \\
\cline { 2 - 3 } & Volumen & capacidad para operar a varios niveles \\
\cline { 2 - 3 } & Material & Capacidad para manejar variaciones en las materias primas \\
\cline { 2 - 3 } & Innovación & capacidad para introducir nuevos productos \\
\hline \multirow{2}{*}{ Entrega } & Rapidez & habilidad para ofrecer productos rápidamente \\
\cline { 2 - 3 } & Oportunidad & Habilidad para ofrecer productos en el momento deseado por el cliente \\
\hline \multirow{2}{*}{ Servicio } & Posventa & Habilidad de respuesta a los requerimientos posventa \\
\cline { 2 - 3 } & Requerimientos & Habilidad para satisfacer las necesidades del cliente \\
\hline Medio \\
ambiente & Proceso & Cumplimiento de normas ambientales \\
\cline { 2 - 3 } & Producto & Productos que respeten el medio ambiente. \\
\hline
\end{tabular}

El estudio se enfocó en las medianas y grandes empresas del sector industrial-metalmecánico. En razón a que estas empresas tienen mayores capacidades de infraestructura para la exportación de sus productos. Para efectos de la clasificación de las empresas, se utilizó de manera informal como único criterio el número de empleados. De este modo, se entiende en este estudio mediana empresa, aquella que tiene una planta de personal entre cincuenta y uno (51) y doscientos (200) trabajadores. Y gran empresa, a la que tiene más de doscientos empleados. Para el caso de la investigación que soporta los resultados a presentar y considerando la emergencia de salud ocasionada por el Covid-19 en el año 2020, se pudo realizar un trabajo de campo con 13 empresas de la región, de un total de 27 existentes. Ahora bien, partiendo del modelo de evaluación, resultado de un constructo teórico, se realizó la validación a partir del análisis de estructuras de correlación, tal como se hizo en los trabajos de Marulanda et al., (2015) y Castro et al., (2015).

\section{RESULTADOS}

El primer paso consistió en realizar el análisis de normalidad multivariante, con el propósito de establecer la asimetría del modelo, la cual presentó estructuras no normales, por lo que se procedió a realizar una transformación de datos con el que pudieran hacer los análisis pertinentes. Seguidamente, se utilizó el análisis de correlación de Pearson, el cual presentó los resultados observados en la tabla 2.Los hallazgos presentados en la tabla 2 permiten validar el modelo de análisis de la estrategia de operaciones para las empresas medianas y grandes del sector metalmecánico del triángulo del café de Colombia, con 7 categorías, 21 variables de análisis y 33 indicadores. Dicho modelo se soporta en los planteamientos teóricos, producto de la experiencia reconocida en una realidad empresarial propia de esta región de Colombia, es decir, con unas connotaciones específicas, un contexto particular y unas necesidades propias de crecer y avanzar en su desarrollo para insertarse en un mundo globalizado, el cual exige retos, dinámicas y soluciones que permitan mejorar los procesos de exportación de sus productos a otros países.

Posteriormente, con el fin de corroborar que las estructuras definidas en el modelo, en el marco de las categorías y variables que tienen relación, se realizó el análisis de correlación entre variables costos y calidad, flexibilidad y servicios, innovación y medio ambiente. Para el efecto, los resultados se pueden observar en las tablas 3,4 y 5 . Con los datos obtenidos en la tabla 3, más del $30 \%$ de los indicadores están por encima de 0,5 , lo cual hace posible inferir que existe una relación significativa entre los bajos costos y la calidad. 


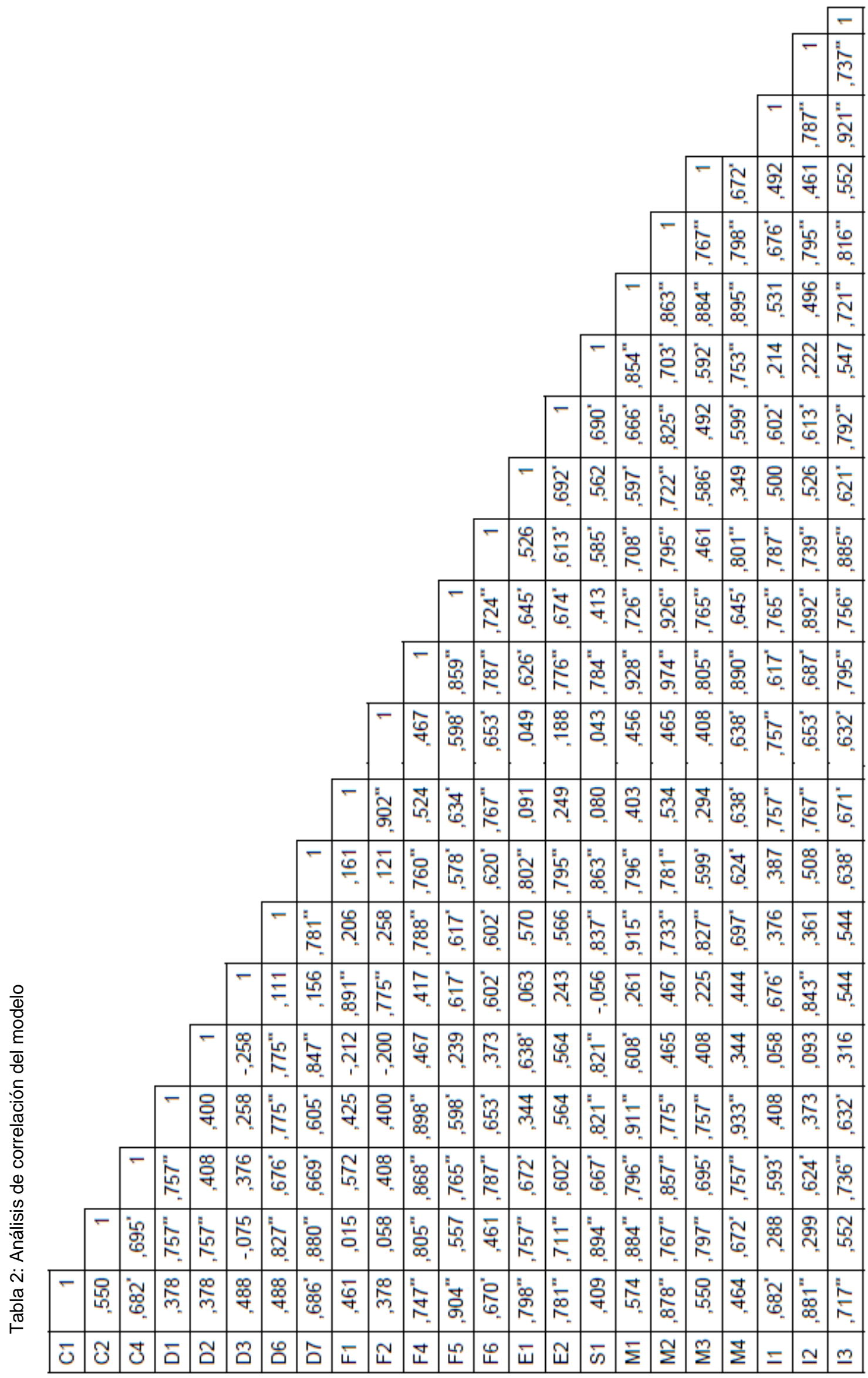

Cabe precisar que, la correlación estadística constituye una técnica estadística que nos indica si dos variables están relacionadas o no. Dicha correlación es medida por lo que se denomina coeficiente de correlación ( $r$ ). Su valor numérico varía de 1,0 a -1,0. En general, $r>0$ indica una relación positiva y $r<0$ indica una relación 
negativa, mientras que $r=0$ indica que no hay relación (o que las variables son independientes y no están relacionadas). Aquí, $r=1,0$ describe una correlación positiva perfecta y $r=-1,0$ describe una correlación negativa perfecta. Estos resultados están alineados con los trabajos de: (Zambrano et al., 2018), quienes concluyeron que la calidad contribuye a la satisfacción de las necesidades de los clientes con los mínimos costos, sobre todo si el trabajo es desarrollado adecuadamente en todas las etapas del proceso de creación de valor (producción, comercialización y administración) y (Telles et al., 2017), quienes explican que la gestión de calidad es una estrategia importante para empresas de cualquier sector, pues está directamente vinculada a la competitividad y la rentabilidad de las mismas. Ahora bien, en cuanto a la flexibilidad y los servicios, los resultados de la correlación se pueden observar en la tabla 4.

Tabla 3: Correlación costos-calidad. *. La correlación es significativa en el nivel 0,05 ( 2 colas). ${ }^{* *}$. La correlación es significativa en el nivel 0,01 (2 colas).

\begin{tabular}{|c|c|c|c|c|c|c|c|c|c|c|c|}
\hline $\mathrm{C} 1$ & 1 & & & & & & & & & & \\
\hline C2 & ,550 & 1 & & & & & & & & & \\
\hline C3 & ,452 &, $905^{\star \star}$ & 1 & & & & & & & & \\
\hline $\mathrm{C} 4$ & ,682* &, $695^{*}$ & ,487 & 1 & & & & & & & \\
\hline D1 & ,378 &, $757^{\star *}$ & ,478 &, $757^{* *}$ & 1 & & & & & & \\
\hline D2 & ,378 &, $757^{* *}$ &, $837^{* *}$ &, 408 & ,400 & 1 & & & & & \\
\hline D3 & ,488 &,- 075 &,- 309 & ,376 & ,258 &,- 258 & 1 & & & & \\
\hline D4 & ,255 &, $746^{\star *}$ & $806^{* *}$ &, 510 &, $674^{*}$ &, $674^{*}$ &,- 174 & 1 & & & \\
\hline D5 & ,255 & ,275 &,- 161 &, 510 &, $674^{*}$ &,- 135 &, 522 &,- 091 & 1 & & \\
\hline D6 & ,488 &, $827^{\star *}$ &, $617^{*}$ &, $676^{*}$ &, $775^{* *}$ &, $775^{\star *}$ & ,111 & ,522 & ,522 & 1 & \\
\hline D7 & $686^{*}$ & $880^{* *}$ & $868^{* *}$ &, $669^{*}$ & $605^{*}$ & $847^{* *}$ &, 156 &, $734^{* *}$ &, 082 &, $781^{* *}$ & 1 \\
\hline
\end{tabular}

Tabla 4: Correlación flexibilidad-servicios. ${ }^{* *}$. La correlación es significativa en el nivel 0,01 (2 colas). *. La correlación es significativa en el nivel 0,05 (2 colas).

\begin{tabular}{|l|r|r|r|r|r|r|r|r|r|r|r|r|}
\hline F1 & 1 & & & & & & & & & & & \\
\hline F2 &, $902^{* *}$ & 1 & & & & & & & & & & \\
\hline F3 &, $850^{* *}$ &, $757^{* *}$ & 1 & & & & & & & & & \\
\hline F4 &, 524 &, 467 &, 554 & 1 & & & & & & & & \\
\hline F5 &, $634^{*}$ &, $598^{*}$ &, $765^{* *}$ &, $859^{* *}$ & 1 & & & & & & & \\
\hline F6 &, $767^{* *}$ &, $653^{*}$ &, $624^{*}$ &, $787^{* *}$ &, $724^{* *}$ & 1 & & & & & & \\
\hline F7 &, 444 &, 239 &, 348 &, $730^{* *}$ &, 571 &, $892^{* *}$ & 1 & & & & & \\
\hline F8 &, $650^{*}$ &, $635^{*}$ &, $607^{*}$ &, $928^{* *}$ &, $867^{* *}$ &, $888^{* *}$ &, $759^{* *}$ & 1 & & & & \\
\hline F9 &, $767^{* *}$ &, $653^{*}$ &, $624^{*}$ &, $787^{* *}$ &, $724^{* *}$ & $1,000^{* *}$ &, $892^{* *}$ &, $888^{* *}$ & 1 & & & \\
\hline S1 &, 080 &, 043 &,- 013 &, $784^{* *}$ &, 413 &, $585^{*}$ &, $723^{* *}$ &, $686^{*}$ &, $585^{*}$ & 1 & & \\
\hline S2 &, 042 &, 158 &, 092 &, $738^{* *}$ &, $661^{*}$ &, 369 &, 378 &, $717^{* *}$ &, 369 &, $684^{*}$ & 1 & \\
\hline S3 &,- 254 &,- 239 &,- 348 &, 558 &, 286 &, 279 &, 500 &, 433 &, 279 &, $827^{* *}$ &, $756^{* *}$ & 1 \\
\hline
\end{tabular}

Con los datos obtenidos en la tabla 4 , más del $30 \%$ de los indicadores están por encima de 0,5 , lo cual hace posible inferir que existe una relación significativa entre la flexibilidad y los servicios. Estos resultados están alineados con los trabajos de: (Cruz y Badii, 2004), quienes sostienen que las empresas requieren implementar diferentes estrategias, tales como: 1) disminuir costos de producción, 2) incrementar la calidad, e 3) incrementar la flexibilidad y tiempo de respuesta; (Arboleda y Rubiano, 2017) concluyen que la mayor capacidad de respuesta en el servicio al cliente se da en la medida en que se puedan alcanzar ciclos más cortos en la fabricación por la introducción de pequeños lotes de producción. En cuanto a la innovación y el medio ambiente, los resultados de la correlación se pueden observar en la tabla 5 . En la tabla, ${ }^{* *}$ significa que la correlación es significativa en el nivel 0,01 (2 colas); ${ }^{*}$ indica que la correlación es significativa en el nivel 0,05 ( 2 colas). Con los datos obtenidos en la tabla 5 , más del $30 \%$ de los indicadores están por encima de 0,5 , lo cual permite inferir que existe una relación significativa entre la innovación y el medio ambiente.

Tabla 5: Correlación innovación - medio ambiente.

\begin{tabular}{|c|c|c|c|c|c|c|c|}
\hline M1 & 1 & & & & & & \\
\hline M2 &, $863^{* *}$ & 1 & & & & & \\
\hline M3 &, $884^{* *}$ &, $767^{* *}$ & 1 & & & & \\
\hline M4 &, $895^{* \star}$ &, $798^{* *}$ & ,672* & 1 & & & \\
\hline M5 &, $831^{* *}$ &, $817^{* *}$ &, $603^{*}$ & $738^{* *}$ & 1 & & \\
\hline 11 &, 531 &, $676^{\star}$ & ,492 &, $586^{*}$ & ,535 & 1 & \\
\hline 12 & ,496 &, $795^{* *}$ & ,461 &, 526 & ,528 &, $787^{* *}$ & 1 \\
\hline 13 &, $721^{\star *}$ &, $816^{* *}$ & ,552 &, $776^{* *}$ &, $741^{* *}$ &, $921^{\star *}$ &, $737^{* *}$ \\
\hline
\end{tabular}

Estos resultados están alineados con los trabajos de: (Bernal y Blanco, 2017), quienes concluyeron que la innovación por diseño es una cuestión de sistemas que no se limita al producto, al proceso o al marketing 
para satisfacer las necesidades de los clientes, sino que abarca todo el sistema empresarial, social, cultural, tecnológico y medioambiental; y (Ramírez, 2017), quien explica que es necesario considerar los dilemas éticos que plantean la innovación y el respeto por el medio ambiente frente a la necesidad empresarial de ser productivo y promover su crecimiento.

\section{DISCUSIÓN FINAL}

La estrategia de operaciones se ha fundamentado básicamente en cuatro o cinco prioridades competitivas según la teoría revisada y la experiencia de los autores. Para el desarrollo del modelo presentado en este documento en relación con el análisis de la estrategia de operaciones de las empresas exportadoras del sector metalmecánico del triángulo del café de Colombia, se consideraron prioridades como el costo, la calidad, la flexibilidad, la entrega, el servicio, el medio ambiente y la innovación, como una forma integral de valoración que implica un enfoque sistémico y holístico. Esto desde una perspectiva integral que acoge la situación propia del contexto empresarial del estudio y de las características específicas de este tipo de empresas en la región y en el país, lo que hace necesariamente que los resultados son propios de una condición poblacional, pero que se pueden replicar en otro sector empresarial y/o en otras regiones de Colombia. El uso de la herramienta de análisis de correlación permitió validar con resultados altamente significativos que el modelo está construido con las prioridades competitivas adecuadas para el sector y tipo de empresas seleccionadas, logrando definir al final un modelo de análisis de estrategia de operaciones compuesto por 7 categorías, 21 variables de análisis y 33 indicadores. De este modo, el desarrollo de la estrategia de operaciones requiere de la integración de cada una de las prioridades competitivas, desde un enfoque integral y considerando el contexto específico de las empresas, así como de marcos de referencia conceptuales y de las condiciones propias del sector exportador evaluado.

\section{CONCLUSIONES}

De acuerdo al trabajo presentado y a los resultados obtenidos, se pueden plantear las siguientes conclusiones principales: 1) La dinámica de gestión de la estrategia de operaciones de las empresas exportadoras del sector metalmecánico del triángulo del café de Colombia, requiere de una integración acorde a sus necesidades empresariales y del entorno, en este sentido es necesario considerar la conceptualización existente y la realidad empresarial actual. 2. El uso de herramientas estadística para la validación de un constructo teórico, partiendo de los marcos de referencias y el análisis de expertos, permitió crear un modelo de análisis de estrategia de operaciones compuesto por 7 categorías y 21 variables adecuadas para las empresas exportadoras del sector metalmecánico del triángulo del café de Colombia

\section{AGRADECIMIENTOS}

Este trabajo es parte de los resultados del proyecto de investigación titulado "Desarrollo de un modelo para el análisis de la estrategia de operaciones de las empresas exportadoras del sector metalmecánico del eje cafetero", que fue registrado y financiado por la Dirección de Investigación de la Universidad Nacional de Colombia sede Manizales, con código Hermes 45325.

\section{REFERENCIAS}

Andrade, A.M., Del Río, C.A., y Alvear, D.L., Estudio de tiempos y movimientos para incrementar la eficiencia en una empresa de producción de calzado, https://dx.doi.org/10.4067/S0718-07642019000300083, Información Tecnológica, 30(3), 83-94 (2019)

Arafa, A., y ElMaraghy, W.H., Manufacturing strategy and enterprise dynamic capability, https://doi.org/10.1016/j.cirp.2011.03.051, CIRP Annals, 60(1), 507-510 (2011)

Arboleda, J., y Rubiano, F.M., Modelo propuesto para la implementación de la metodología SMED en una empresa de alimentos de Santiago de Cali, https://doi.org/10.29097/2011-639X.85, Revista de Investigación, 10(2), 103-117 (2017)

Avella, L., Fernández, E., y Vázquez, C.J., Análisis de las estrategias de fabricación como factor explicativo de la competitividad de la gran empresa industrial Española, Cuadernos de Economía y Dirección de la Empresa, 4, 235-258 (1999)

Badri, M.A., Davis, D., y Davis, D., Operations strategy, environmental uncertainty and performance: a path analytic model of industries in developing countries, https://doi.org/10.1016/S0305-0483(99)00041-9, Omega, 28(2), 155-173 (2000)

Bernal-Torres, C.A., y Blanco-Valbuena, C.E., Innovación por diseño y su relación con las variables del entorno en una muestra de empresas en Bogotá-Colombia, http://dx.doi.org/10.4067/S0718-07642017000400017, Información Tecnológica, 28(4), 145-156 (2017)

Castro, M.A.D., Baltar, V.T., Selem, S.S.D.C., Marchioni, D.M.L., y Fisberg, R.M., Empirically derived dietary patterns: interpretability and construct validity according to different factor rotation methods, https://doi.org/10.1590/0102311X00070814, Cadernos de Saude Publica, 31(2), 298-310 (2015) 
Chatha, K.A., y Butt, I., Themes of study in manufacturing strategy literature, https://doi.org/10.1108/IJOPM-07-20130328, International Journal of Operations \& Production Management, 35(4), 604-698 (2015)

Cruz, J., y Badii, M.H., SMED: El camino a la flexibilidad total, Innovaciones de Negocios, 1(2) 277-283 (2004)

Dangayach G.S., y Deshmukh, S.G., Manufacturing strategy literature review and some issues, https://doi.org/10.1108/01443570110393414, Int. J. of Operation \& Production Management, 21(7), $884-932$ (2001)

De La Hoz, E., González, A.L., y Santana, A. Metodología de medición del potencial exportador de las organizaciones empresariales, http://dx.doi.org/10.4067/S0718-07642016000600003, Información Tecnológica, 27(6), 11-18 (2016)

Díaz, G., y Martín, M.L., Un análisis de las configuraciones genéricas de la estrategia de producción de empresas industriales españolas, https://doi.org/10.1016/S1138-5758(07)70094-4, Cuadernos de Economía y Dirección de la Empresa, 10(32), 149-175 (2007)

Göleç, A., A relationship framework and application in between strategy and operational plans for manufacturing industry, https://doi.org/10.1016/j.cie.2014.10.007, Computers \& Industrial Engineering, 86, 83-94 (2015)

Größler, A., The development of strategic manufacturing capabilities in emerging and developed markets, https://doi.org/10.1007/s12063-010-0033-3, Operations Management Research, 3, 60-67 (2010)

Guerra-Borges, A., Regionalismo y multilateralismo en su laberinto, Problemas del Desarrollo, Revista Latinoamericana de Economía, 39(152), 11-28 (2008)

Korsakienè, R., Liučvaitienè, A., Bužavaitè, M., y Šimelytè, A., Intellectual capital as a driving force of internationalization: a case of Lithuanian Smes, http://doi.org/10.9770/jesi.2017.4.4(8), The International Journal Entrepreneurship and Sustainability Issues, 4(4), 502-515 (2017)

León, F. G. J., Administración de operaciones: análisis de las estrategias de operaciones en las empresas como elemento clave para la competitividad, http://doi.org/10.23857/pc.v5i10.1832, Polo del Conocimiento, 5(10), 551-559 (2020)

Lobo, G., Pinheiro, E., y Gouvea, S.E., Efficiency frontier identification based on operations strategy: a retrospective analysis of leading authors, https://doi.org/10.1016/j.promfg.2020.01.431, Procedia Manufacturing, 39, 775-785, (2019a)

Lobo, G., Pinheiro de Lima, E., Aken, E., y Gouvea da Costa, S., Efficiency frontier identification on the context of operations strategy: a study on representative constructs and variables, https://doi.org/10.1016/j.promfg.2020.01.436, Procedia Manufacturing, 39, 745-755, (2019b)

Martínez-Sánchez, Á., Vela, M., Abella S., y Gorgemans, S., Flexibility and innovation: moderator effects of cooperation and dynamism, https://doi.org/10.1108/PR-12-2017-0397, Personnel Review, 48(6), 1548-1564, (2019)

Marulanda, C.E., Giraldo, J.A., y Serna, H.M., Knowledge management assessment model for information technology SMEs, http://dx.doi.org/10.17230/ad-minister.26.2, AD-Minister, 26, 17-39 (2015)

Patil, P.P., Narkhede, B.E., y Akarte, M.M., Pattern of manufacturing strategy implementation and implications on manufacturing levers and manufacturing outputs and business performance, https://doi.org/10.1504/IJICBM.2015.068162, Int. J. of Indian Culture and Business Management, 10(2), 157-177 (2015)

Ramírez, J.V., El sentido ético en la responsabilidad social: economía, innovación y medio ambiente, Ensayos de Economía, 50, 15-36 (2017)

Sáenz, R., Operaciones: concepto, sistema, estrategia y simulación, Revista Latinoamericana de Administración, 34, 124 (2005)

Telles, L.B., Bittencourt, J.V.M., y Pitta, C.S.R., Herramientas y sistema de costos aplicados a la gestión de la calidad en el agronegocio, Interciencia, 42(5), 301-306 (2017)

Thürer, M., Godinho, M., Stevenson, M., y Fredendall, L.D., Small manufacturers in Brazil: competitive priorities vs. capabilities, https://doi.org/10.1007/s00170-014-6042-x, The International Journal of Advanced Manufacturing Technology, 74, 1175-1185 (2014)

Wheelwright, S.C., Reflecting corporate strategy in manufacturing decisions, https://doi.org/10.1016/00076813(78)90032-0, Business horizons, 21(1), 57-66 (1978)

Zambrano, M.I., Véliz, V.F., Armada, E., y López, M., Los costos de calidad: su relación con el sistema de costeo ABC, Cofin Habana, 12(2), 179-189 (2018)

Zhan, Y., Tan, K.H., Ji, G., y Tseng, M., Sustainable Chinese manufacturing competitiveness in the 21st century: green and lean practices, pressure and performance, https://doi.org/10.1080/0951192X.2016.1268721, Int. J. of Computer Integrated Manufacturing, 31(6), 523-536 (2018)

Zhao X., y Lee, T., Developments and emerging research opportunities in operations strategy and supply chain management, https://doi.org/10.1016/j.ijpe.2008.12.010, Int. J. of Production Economics, 120(1), 1-4 (2009) 